\title{
Oxidative stress as a biomarker for Alzheimer's disease
}

\author{
Fabrice Collin*,1,2, Clémence Cheignon ${ }^{1,2,3}$ \& Christelle Hureau ${ }^{1,2}$ \\ ${ }^{1}$ Laboratoire de Chimie de Coordination (LCC), CNRS UPR 8241, 205 route de Narbonne, 31062 Toulouse Cedex 09, France \\ ${ }^{2}$ Université de Toulouse, UPS, INPT, 31077 Toulouse, France \\ ${ }^{3}$ Institut des Biomolécules Max Mousseron (IBMM), Université de Montpellier, 34095 Montpellier Cedex 5, France \\ * Author for correspondence: fabrice.collin@univ-tlse3.fr
}

\section{'In the search for oxidative stress biomarkers, should the specificity include both AD and oxidative stress?"}

First draft submitted: 26 December 2017; Accepted for publication: 8 January 2018; Published online: 13 February 2018

Keywords: Alzheimer's disease $\bullet$ amyloid beta peptide $\bullet$ lipid peroxidation $\bullet$ oxidative stress $\bullet$ peripheral biofluids - tau protein

It is estimated that Alzheimer's disease (AD) is striking 1 in 20 over age 65, which yielded to 23-35 million people worldwide in 2015. The number of AD victims is expected to almost double every 20 years, thus placing the national healthcare systems under dramatically increasing pressure. This clearly underlines the need to find early biomarkers and efficient medical treatments for $\mathrm{AD}$, both implying a better understanding of the mechanism from the origin of the disease to the death of the neurons. Oxidative damages on neuronal lipids and proteins, in particular, are an important feature of $\mathrm{AD}$ and a link with oxidative stress [1]. The latter can have different origins, but the overproduction of reactive oxygen species (ROS) is considered as a major contribution. Loosely bound metal ions (copper and iron), present in the brain and at high concentration in senile plaques of AD patients, may bind to the amyloid beta peptide $(\mathrm{A} \beta)$ thus catalyzing very efficiently the production of ROS (in particular, the oligomeric forms of $A \beta$, known as being the most toxic). This represents one of the source for ROS production in brain, a second one being mitochondria dysfunction.

\section{What are the biomarker candidates?}

Along with cognitive testing, $A \beta 42$, total and phosphorylated tau levels are currently the recommended biomarkers in the cerebrospinal fluid (CSF) for using in clinic to establish a diagnosis of AD [2]. Biomarkers of oxidative stress in $\mathrm{AD}$ can either be directly produced after an attack of biomolecules by ROS, result from a reaction between a primary oxidation product - such as 4-hydroxynonenal - and a biomolecule, or be a metabolite that is indirectly upregulated or downregulated by oxidative stress. Thus, usual markers of protein, lipid, DNA and RNA oxidation are associated with $\mathrm{AD}$ : carbonylation of proteins, lipid peroxidation, oxidation of nuclear and mitochondrial DNA bases (resulting in 8-oxo-2-dehydroguanine, 8-hydroxyadenine and 5-hydroxyuracil production) and oxidation of mRNA [3]. Lipid peroxidation is involved in disruption of cell membrane integrity and also in the production of 4-hydroxynonenal that is further able to react with proteins (lysine and cysteine residues), as for example, tau or LRP1. Oxidation of the latter would be responsible for the disruption of A $\beta$ clearance in the brain [4], leading to its accumulation. Oxidation of cholesterol into oxysterols is also reported to play a role in amyloidogenesis, in particular, 24- and 27-hydroxycholesterols [5]. Some of other commonly reported oxidative stress biomarkers of AD are ApoE genotype, malondialdehyde, 8-hydroxy-2'-dehydroguanosine, coenzyme Q10, GSH/GSSG, superoxide dismutase, hydrogen peroxide, glutathione peroxidase and reductase and isoprostanes (see [6] for a review of AD studies). Finally, as metallated $A \beta$ is one of the significant source of ROS production in the brain, the peptide itself undergoes the ROS attack and the oxidized $A \beta$ peptide could thus appear as a very specific oxidative stress biomarker for $\mathrm{AD}$. 


\section{Which ones would be relevant in the context of in vivo sampling procedures?}

The relevance of a biomarker for any pathology would lie in three important points: it must be associated as specifically as possible with a particular pathology; it must be easy to detect and quantify, by affordable and robust analytical methodologies; and finally, it must be present in peripheral tissue or fluid - meaning accessible by noninvasive techniques. In the context of $\mathrm{AD}$, a neurodegenerative pathology, the last point is the first to take into account since potential biomarkers specifically present in the brain can only be quantified postmortem in brain tissue. Actually, diagnosis can be made by the use of analytical methodologies based on brain imaging - such as magnetic susceptibility [7] or positron emission tomography [8], for example, used for $A \beta$ and tau pathologies. However, they usually require sophisticated equipment and are expensive. In clinical and preclinical stages, AD diagnosis is based on the presence of $A \beta$ and tau in the CSF, which require an invasive sampling procedure. The future of biomarkers discovery for $\mathrm{AD}$ thus seems to lie in developing strategies that focus on searching biomarkers in easily available tissues, meaning peripheral biofluids such as blood. Many efforts are currently done by research groups worldwide for finding early AD biomarkers in blood (including plasma, serum, platelets, red blood cells and lymphocytes). The broadly accepted CSF biomarkers are not good for blood since they show either no significant change between $\mathrm{AD}$ patient and healthy controls or a considerable variability between studies [9]. Systematic review of AD studies in the literature may help in finding relevant candidates [6], and promising research strategies could focus on lipid peroxidation - in particular, lisophosphatic acid C18:2 [10] - and high levels of isoprostanes and neuroprostanes, found in plasma, but also urine and CSF. However, no consensus has been found yet.

\section{The quest for an early \& specific biomarker of AD}

Oxidative stress being an early event in $\mathrm{AD}$ etiology, one can assume that associated biomarkers could represent an interesting opportunity in terms of early diagnosis of AD. Markers of oxidative stress were suggested as consistent elements of blood-based signatures in AD [10]. Searching for good candidates is often based on AD studies in the literature and/or statistical analysis. To date, none of the suggested biomarkers were validated by large cohort studies at preclinical/clinical stage and associated with $\mathrm{AD}$ progression. One reason is because the search for oxidative stress biomarkers for $\mathrm{AD}$ is still an emerging field, or actually was not the first way explored by researchers. The major pitfall is that oxidative stress is one of the responses to inflammation and is also commonly induced by various pathologies (such as cancer, atherosclerosis or diabetes), which could share some of the potential biomarkers. For instance, lipid peroxidation and malondialdehyde are also biomarkers of cancer. The current diagnosis of AD is established, biochemically, by $A \beta$, total and phosphorylated tau levels, three specific biomarkers. In the search for oxidative stress biomarkers, should the specificity include both AD and oxidative stress? Yes probably, and this reduce the possibilities. In this perspective, one can wonder if oxidation levels of the A $\beta$ peptide, the AD's closely associated molecule, could be of major interest. Circulating $A \beta$ has been detected in plasma, along with oligomeric forms that could be considered as potential biomarkers [11]. As the latter are known as the most toxic forms of $A \beta$ (compared with monomer or fibrils), in particular, because they are associated with ROS production, it can be assumed that oxidized $A \beta$ species would also be present. The development of antibodies directed against oxidized $A \beta$ would help in such a quest, although it is challenging since expected levels of circulating oxidized $A \beta$ should be very low, especially at the beginning of $\mathrm{AD}$ progression. However, while continuing to search for other oxidative stress biomarkers, this quest should be very attractive.

Financial \& competing interests disclosure

The authors have no relevant affiliations or financial involvement with any organization or entity with a financial interest in or financial conflict with the subject matter or materials discussed in the manuscript. This includes employment, consultancies, honoraria, stock ownership or options, expert testimony, grants or patents received or pending, or royalties.

No writing assistance was utilized in the production of this manuscript.

\section{References}

1 Cheignon C, Tomas M, Bonnefont-Rousselot D, Faller P, Hureau C, Collin F. Oxidative stress and the amyloid beta peptide in Alzheimer's disease. Redox Biol. 14, 450-464 (2018).

2 Olsson B, Lautner R, Andreasson U et al. CSF and blood biomarkers for the diagnosis of Alzheimer's disease: a systematic review and meta-analysis. Lancet Neurol. 15(7), 673-684 (2016).

3 Butterfield DA, Reed T, Newman SF, Sultana R. Roles of amyloid beta-peptide-associated oxidative stress and brain protein modifications in the pathogenesis of Alzheimer's disease and mild cognitive impairment. Free Radic. Biol. Med. 43(5), 658-677 (2007). 
4 Owen JB, Sultana R, Aluise CD et al. Oxidative modification to LDL receptor-related protein 1 in hippocampus from subjects with Alzheimer disease: implications for A $\beta$ accumulation in AD brain. Free Radic. Biol. Med. 49(11), 1798-1803 (2010).

5 Heverin M, Bogdanovic N, Lutjohann D et al. Changes in the levels of cerebral and extracerebral sterols in the brain of patients with Alzheimer's disease. J. Lipid Res. 45(1), 186-193 (2004).

6 Garcia-Blanco A, Baquero M, Vento M, Gil E, Bataller L, Chafer-Pericas C. Potential oxidative stress biomarkers of mild cognitive impairment due to Alzheimer disease. J. Neurol. Sci. 373, 295-302 (2017).

7 O'Callaghan J, Holmes $\mathrm{H}$, Powell $\mathrm{N}$ et al. Tissue magnetic susceptibility mapping as a marker of tau pathology in Alzheimer's disease. Neuroimage 159, 334-345 (2017).

8 Marcus C, Mena E, Subramaniam RM. Brain PET in the diagnosis of Alzheimer's disease. Clin. Nucl. Med. 39(10), e413-e426 (2014).

9 Wojsiat J, Laskowska-Kaszub K, Mietelska-Porowska A, Wojda U. Search for Alzheimer's disease biomarkers in blood cells: hypotheses-driven approach. Biomark. Med. 11(10), 917-931 (2017).

10 de Leeuw FA, Peeters CFW, Kester MI et al. Blood-based metabolic signatures in Alzheimer's disease. Alzheimers Dement. (Amst.) 8 , 196-207 (2017).

11 Wang MJ, Yi S, Han JY et al. Oligomeric forms of amyloid-beta protein in plasma as a potential blood-based biomarker for Alzheimer's disease. Alzheimers Res. Ther. 9(1), 98 (2017). 
